Sawtooth control in ITER using ion cyclotron resonance heating

This article has been downloaded from IOPscience. Please scroll down to see the full text article.

2011 Plasma Phys. Control. Fusion 53124003

(http://iopscience.iop.org/0741-3335/53/12/124003)

View the table of contents for this issue, or go to the journal homepage for more

Download details:

IP Address: 128.178.125.184

The article was downloaded on 26/09/2012 at 11:22

Please note that terms and conditions apply. 


\title{
Sawtooth control in ITER using ion cyclotron resonance heating
}

\author{
I T Chapman ${ }^{1}$, J P Graves ${ }^{2}$, T Johnson ${ }^{3}$, O Asunta ${ }^{4}$, P Bonoli ${ }^{5}$, M Choi ${ }^{6}$, \\ E F Jaeger ${ }^{7}$, M Jucker ${ }^{2}$ and O Sauter ${ }^{2}$ \\ ${ }^{1}$ Euratom/CCFE Fusion Association, Culham Science Centre, Abingdon, OX14 3DB, UK \\ 2 CRPP, Association EURATOM/Confédération Suisse, EPFL, 1015 Lausanne, Switzerland \\ ${ }^{3}$ EURATOM-VR Association, EES, KTH, Stockholm, Sweden \\ ${ }^{4}$ Association EURATOM-Tekes, Aalto University, Department of Applied Physics, \\ PO Box 14100 FI-00076 AALTO, Finland \\ 5 Plasma Science and Fusion Center, MIT, Cambridge, Massachusetts 02139-4307, USA \\ ${ }^{6}$ General Atomics, PO Box 85608, San Diego, CA 92186, USA \\ ${ }^{7}$ Oak Ridge National Laboratory, Oak Ridge, Tennessee 37831-8071, USA \\ E-mail: ian.chapman@ccfe.ac.uk
}

Received 24 June 2011, in final form 7 July 2011

Published 14 November 2011

Online at stacks.iop.org/PPCF/53/124003

\begin{abstract}
Numerical modelling of the effects of ion cyclotron resonance heating (ICRH) on the stability of the internal kink mode suggests that ICRH should be considered as an essential sawtooth control tool in ITER. Sawtooth control using ICRH is achieved by directly affecting the energy of the internal kink mode rather than through modification of the magnetic shear by driving localized currents. Consequently, ICRH can be seen as complementary to the planned electron cyclotron current drive actuator, and indeed will improve the efficacy of current drive schemes. Simulations of the ICRH distribution using independent $\mathrm{RF}$ codes give confidence in numerical predictions that the stabilizing influence of the fusion-born alphas can be negated by appropriately tailored minority ${ }^{3} \mathrm{He}$ ICRH heating in ITER. Finally, the effectiveness of all sawtooth actuators is shown to increase as the $q=1$ surface moves towards the manetic axis, whilst the passive stabilization arising from the alpha and NBI particles decreases.

(Some figures in this article are in colour only in the electronic version)
\end{abstract}

\section{Introduction}

Sawtooth control remains an important unresolved issue for baseline scenario operation of ITER. Since the monotonic $q$-profile of ELMy H-mode plasmas have a large radial position of $q=1, r_{1}$, with low magnetic shear at the $q=1$ surface, $s_{1}=r_{1} \mathrm{~d} q / \mathrm{d} r$, these plasmas are 
expected to be unstable to the internal kink mode. The energetic trapped fusion-born $\alpha$-particles are predicted to lead to significant stabilization of the internal kink mode [1-3], resulting in very long sawtooth periods. However, such long sawtooth periods have been observed to result in triggering of neoclassical tearing modes (NTMs) at lower plasma $\beta$ [4-6]. These NTMs can, in turn, significantly degrade plasma confinement. Consequently, there is an urgent need to assess whether sawtooth control will be achievable in ITER and how much power is required from the actuators at our disposal to attain an acceptable sawtooth period. Our understanding of internal kink mode stability that underlies sawteeth has improved significantly in recent times through a combination of analytic understanding, experimental verification and detailed modelling, as reviewed in [7].

For many years it has been known that trapped energetic particles result in strong stabilization of sawteeth. However, passing fast ions can also significantly influence sawtooth behaviour. For highly energetic ions, the radial drift motion becomes comparable to the radial extent of the kink mode. In this regime, the kinetic contribution to the mode's potential energy (together with a non-convective contribution to the fluid part of $\delta W$ ) becomes increasingly important. When the passing fast ion population is asymmetric in velocity space, there is an important finite orbit contribution to the mode stability. The effect of passing ions is enhanced for large effective orbit widths [8], which is to say, for highly energetic ions (like ion cyclotron resonance heating (ICRH) or N-NBI in ITER) or for a population with a large fraction of barely passing ions (such as ICRH or NBI in JET). Passing fast ions can destabilize the internal kink mode when they are co-passing (i.e. moving in the same direction as the plasma current) and the fast ion distribution has a positive gradient across $q=1$, or when they are counter-passing, but the deposition is peaked outside the $q=1$ surface. This mechanism is described in detail in $[9,10]$. The effect of passing fast ions has been confirmed in NBI experiments in JET [11, 12], ASDEX Upgrade [13] and MAST [14] and using $\mathrm{He}^{3}$ minority ICRH in JET $[15,16]$. By employing $\mathrm{He}^{3}$ minority heating schemes (which are envisaged for ITER ICRF heating), the resultant current drive is negligible [10,17]. Nonetheless, the ICRH can still strongly influence the sawtooth stability, demonstrating that sawtooth control via ICRH can be achieved via a kinetic destabilization mechanism rather than through local modification of the magnetic shear at $q=1$.

An empirical scaling of the sawtooth period that will trigger an NTM in ITER [6] suggests that the 'natural' sawtooth period (i.e. in the absence of active control) predicted by transport modelling [18-20] is approximately at the threshold for NTM seeding for baseline operation at $\beta_{\mathrm{N}}=1.8$. Whilst this means that active sawtooth control is essential, it suggests that sufficient control can be achieved through a relatively small reduction in the sawtooth period. Transport modelling coupled to ray-tracing predictions and using the linear stability thresholds for sawtooth onset from [1] suggests that $13 \mathrm{MW}$ of ECCD from the equatorial launcher could be sufficient to reduce the sawtooth period by $\sim 30 \%$, and this being the case, dropping it below the NTM triggering threshold [21,22]. However, this modelling is predicated upon choosing a natural sawtooth period of $40 \mathrm{~s}$; should the stabilizing contribution from the fusion-born alpha particles and on-axis NBI injection prove to give rise to a significantly longer natural sawtooth period, the ECCD efficacy will be diminished. There are naturally large uncertainties associated in this modelling, and it is prudent to plan to use more than one control actuator in order to reduce this risk. Consequently, in this paper we consider whether ICRH can be used as a complementary control tool together with ECCD in ITER. Since sawtooth control with ECCD works by modifying the local magnetic shear $[23,24]$ whereas ICRH directly affects the fast ion contribution to mode energy, this reduces the risk in relying upon one control actuator. In section 2 the numerical codes used to model the fast ion distributions are described whilst the stability modelling is outlined in section 3. The fast ion distributions in ITER are then 
calculated in section 4 and their effect on kink mode stability is found in section 5 before the implications for ITER are given in section 6.

\section{Energetic particle population modelling tools}

In order to model the neutral beam fast ion distribution, the TransP [25] and Ascot [26, 27] codes have been used. Asсот has been used to model the alpha particle population whilst SELFo [28], SCENIC [29, 30] and a coupling of Aorsa [31] with CQL3D [32] have been used to simulate the ICRH distribution. Finally, the HaGIS drift kinetic code [33] has been employed to study the effect of the various fast ion populations on internal kink stability.

The TRAnsP code was used to simulate the NBI fast ion population since it enables the use of the beam module NuBEAm in a convenient, integrated plasma simulation environment. The NuBEAM module is a Monte Carlo package for time dependent modelling of fast ion species in an axisymmetric tokamak using classical physics. Multiple fast ion species can be present, due to either beam injection of energetic neutral particles deposited as ions in the target plasma, or as a product of nuclear fusion reactions. The model self-consistently handles classical guidingcentre drift orbiting, collisional and atomic physics effects during the slowing down of the fast species population (represented by an ensemble of Monte Carlo model particles). In order to reduce the risk in a result dependent entirely upon the prediction of the NUBEAM module within TrANSP, the AsCOT code has also been used to simulate the NNBI distribution. Ascot [27] is a guiding-centre orbit following Monte Carlo code which integrates the particles' equation of motion in time over a five-dimensional space. Collisions with the background plasma are modelled using Monte Carlo operators allowing an acceleration of collisional time scales and reduced computational time. The alpha particle markers are initialized by the local $\langle\sigma v\rangle_{\mathrm{DT}}$ whereas the beam ions are followed starting from the injector taking into account the beamlet position, direction, beam species, energy, total power and its bi-Gaussian dispersion. The ionization cross-section is calculated at each step using the local temperature and density, and analytic fits from [34]. In addition to thermal fusion reactions, also fusion reactions between the fast NBI particles and thermal plasma particles are included in the Ascot code using the model described in [49].

The ICRH fast ion populations are simulated using SELFO, SCENIC and Aorsa/CQL3D. The SELFo code [28] determines self-consistently the power absorption and the fast ion acceleration by coupling the global wave solver Lion [35] and the Monte Carlo code FIDo [36]. FIDo solves the $3 \mathrm{D}$ orbit averaged kinetic equations, including quasilinear ICRF acceleration from the LION wave field. FIDO accounts for wide guiding-centre orbits, including all possible shapes of banana and potato orbits. LiON does not include the upshift of the parallel wave number. Thus SELFo can be used to treat harmonic heating schemes, but not mode conversion. A limitation in the present version of the FIDO code is the assumption of circular flux surfaces. To minimize the error caused by this assumption, the ITER equilibrium has been mapped so that the poloidal flux function in the outboard midplane $\left\{\psi\left(R, Z=Z_{\text {axis }}\right) \mid R>R_{\text {axis }}\right\}$ is the same in SELFO as in the non-circular ITER equilibrium. Furthermore, the ICRH power is normalized so that the power absorbed per resonant ion is the same.

In order to reduce the uncertainty in simulating the fast ion effects resulting from the distribution produced by SELFO, two further code-packages for simulating the RF-induced energetic ion population have been employed, namely SCENIC and AORSA/CQL3D. The ScENIC integrated code package [29,30] takes an equilibrium from ANIMEC [37], the wave fields and wave numbers from LEMan [38] and iterates with the distribution function evolved by Venus $[39,40]$. These codes are iterated to form a self-consistent solution which can incorporate the anisotropic equilibrium in full 3D geometry. For the equilibrium and wave 
field computations, a bi-Maxwellian distribution is used for the hot minority, allowing for pressure anisotropy and stronger poloidal dependence of the pressure and dielectric tensor. Whereas LEMan is limited to leading order FLR effects, and thus to fundamental harmonic without mode conversion, it computes the wave vectors with the help of an iterative scheme, and can therefore treat correctly upshifted wave numbers without the use of a local dispersion relation.

A similar iterative coupling between the AorsA wave field code [31] and the Fokker-Planck CQL3D code [32] (which neglects orbit widths) has been developed. Aorsa is used to solve the RF wave electric fields assuming a Maxwellian electron distribution and a combination of thermal and energetic ion species. The code includes the upshift effect in parallel wave number with no limitation to high ion cyclotron harmonics heating. The non-thermal ion species distribution is provided as a flux surface bounce-averaged quantity by CQL3D. For nonMaxwellian species Aorsa evaluates the susceptibility directly from the ion distribution. The bounce-averaged diffusion coefficients derived from AORSA wave fields are fed into CQL3D, and in such a way an iterative coupling of the codes allows self-consistent calculations of the power absorption for minority heating scenarios.

Rather than attempting a benchmark between the RF codes, as for instance in [41], here the marker populations from each ICRH code are used to study the effect on the internal kink, and it is this (de)stabilizing effect that is compared. In this way, using three independent platforms to generate the ICRH fast ion distribution function is considered as an error reduction strategy, with the worst case scenario (here, the least destabilizing effect) used to determine the power requirements.

\section{Stability model}

\subsection{Linear stability criteria}

The fundamental trigger of the sawtooth crash is thought to be the onset of an $m=n=1$ internal kink mode. However, the dynamics of this mode are constrained by many factors, including not only the macroscopic drive from ideal MHD, but collisionless kinetic effects related to high energy particles [42] and thermal particles [43] as well as non-ideal effects localized in the narrow layer around $q=1$. A heuristic model predicts that a sawtooth crash will occur when one of three criteria is met $[1,44]$. In the presence of fast ions, two conditions are unlikely to be satisfied since the magnetic drift frequency of the hot ions, $\omega_{\mathrm{dh}}$, will be large and $\delta \hat{W}$ may have a large positive contribution from $\delta \hat{W}_{\mathrm{h}}$. The change in the kink mode potential energy is defined such that $\delta \hat{W}=\delta \hat{W}_{\text {core }}+\delta \hat{W}_{\mathrm{h}}$ where $\delta \hat{W}_{\text {core }}=\delta \hat{W}_{\mathrm{MHD}}+\delta \hat{W}_{\mathrm{KO}}$ and $\delta \hat{W}_{\mathrm{KO}}$ is the change in the mode energy due to the collisionless thermal ions [43], $\delta \hat{W}_{\mathrm{h}}$ is the change in energy due to the fast ions and $\delta \hat{W}_{\mathrm{MHD}}$ is the ideal fluid mode drive [45]. The potential energy is normalized such that $\delta \hat{W} \equiv 4 \delta W /\left(s_{1} \xi_{0}^{2} \epsilon_{1}^{2} R B^{2}\right)$ and $\xi_{0}$ is the plasma displacement at the axis, $\epsilon_{1}=r_{1} / R, R$ is the major radius and $B$ is the magnetic field. When $\delta \hat{W}$ is large and positive, the mode takes the structure of a tearing mode, which is non-ideal and can be weakly unstable. It is assumed that these drift-tearing modes are stabilized by diamagnetic effects, so do not drive sawtooth crashes. When the potential energy is sufficient to drive a resistive kink mode, the sawtooth crash is determined by the domain in which the resistive mode can be destabilized, that is to say when

$$
-c_{\rho} \hat{\rho}<-\delta \hat{W}<\frac{1}{2} \omega_{* \mathrm{i}} \tau_{\mathrm{A}},
$$

where $\tau_{\mathrm{A}}=\sqrt{3} R / v_{\mathrm{A}}$ is the Alfvén time, $c_{\rho}$ is a normalization coefficient of the order of unity that determines the threshold at which the mode is considered to result in a sawtooth 
crash, $\omega_{* \mathrm{i}}$ is the ion diamagnetic frequency, $\hat{\rho}=\rho_{\theta \mathrm{i}} / r_{1}$ and the poloidal ion Larmor radius is $\rho_{\theta \mathrm{i}}=v_{\text {thi }} m_{\mathrm{i}} / e B_{\theta}$ where $B_{\theta}=\mu_{0} I_{\mathrm{p}} / 2 \pi a$ and $v_{\text {thi }}=\left(k T_{\mathrm{i}} / m_{\mathrm{i}}\right)^{1 / 2}$. Typically in the presence of fast ions, the sawtooth crash is triggered by a resistive kink mode when inequality (1) is satisfied. However, it should be noted that the crash can still be triggered by an ideal internal kink if the magnetic shear is sufficiently large that the normalization of $\delta W$ results in a crash. In ITER, $\rho_{\theta \mathrm{i}}$ will be small and $r_{1}$ is expected to be large, meaning that satisfying (1) by increasing $s_{1}$ alone may not be possible if $\delta W$ is large and positive, so it is prudent to find ways to directly reduce the fast ion stabilization.

\subsection{Alpha particle effects on kink mode stability}

The effect of the fast ions on the kink mode stability is tested using the Monte Carlo guidingcentre drift kinetic code HaGis [33]. The equilibrium is calculated with the static fixed-boundary 2D Grad-Shafranov solver HeLENA [46]. The stability of this equilibrium is then tested using the linear MHD code Mishra [47]. The perturbation and equilibrium are then fed into HaGis together with the distribution functions of fast ions from the modelling described in section 2 .

HaGis is a Monte Carlo code which solves the non-linear drift guiding-centre equations of motion. It allows the evolution of a fast ion population to be studied in the presence of electromagnetic perturbations in a toroidal plasma. The HAGIS code has been used extensively for studying the stability of the internal kink mode, successfully replicating experimental signatures of sawtooth behaviour on JET [2,11,15], TEXTOR [12] and ASDEX Upgrade $[13,14]$. This gives confidence in using HaGIs to test the effect of NBI and ICRH ions, though the alpha particle contribution, which is predicted to give rise to the most stabilizing contribution, has not previously been verified against empirical evidence.

In order to try to demonstrate that the numerical modelling can differentiate between the competing effects of NBI and alpha particles, data from the JET deuterium-tritium campaign [48] has been modelled using TRANSP. In the JET D-T campaign, the sawtooth period increased with the tritium fraction. HAGIs modelling suggests that for these relatively low fusion power plasmas, the NBI heating dominates over the effect of the fusion alphas. The tritium fraction dependence arises through a combination of (i) the orbit width scaling with the mass and (ii) the beam tritons taking longer to slow down meaning that the stronger pressure gradient gives a larger $\delta W_{\mathrm{NBI}}$. This is illustrated in figure 1, where the total change in the kink mode potential energy increases with tritium fraction despite the reduction in the alpha particle contribution.

\section{Energetic particle distributions in ITER}

\subsection{Core fast particles}

The distribution of alpha particles has been tested with AscoT with no ferritic inserts, and in the case when there is a $3 \mathrm{D}$ equilibrium field due to the presence of ferritic inserts. In both cases, the alphas are well confined within $\rho \sim 0.6$ and are approximately isotropic.

In order to penetrate the hot, dense plasmas in ITER, neutral deuterium beam energies of the order of $0.5-1.0 \mathrm{MeV}$ are necessary. In this study, the N-NBI is assumed to consist of $1 \mathrm{MeV}$ (D) neutrals from a negative ion-beam system injected in the co-current direction, at a tangency radius of $6 \mathrm{~m}$. This generates a broad beam-driven current profile with a total driven current of 1.2 MA [50]. The beam can be aimed at two extreme (on-axis and off-axis) positions by tilting the beam source around a horizontal axis on its support flange, resulting in $\mathrm{N}-\mathrm{NBI}$ injection in the range of $Z=-0.25$ to $-0.95 \mathrm{~m}[51]$. 


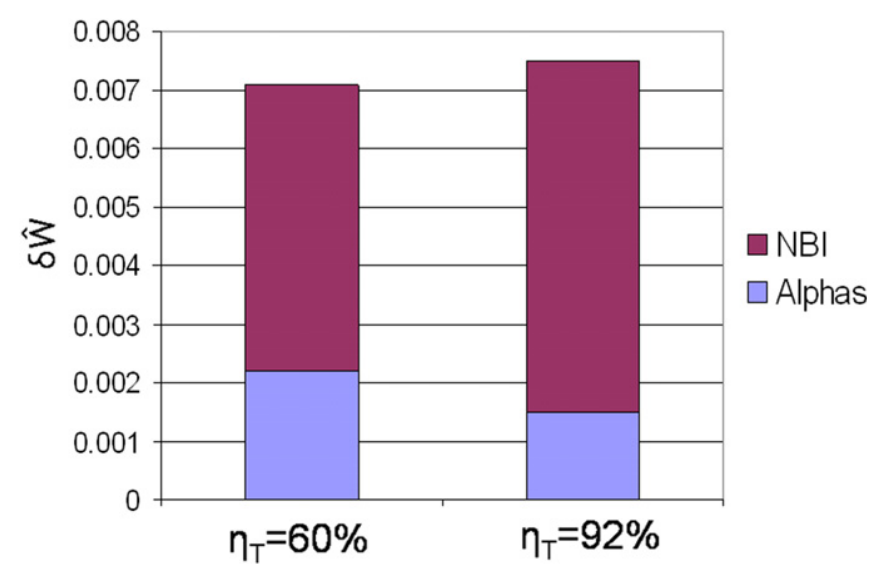

Figure 1. The change in the potential energy of the kink mode modelled by HAGIs for two JET shots with different tritium concentration. When the tritium concentration is high, the beam slowing down time is longer, and so the NBI fast ions are more stabilizing, explaining the longer sawtooth period despite a comparatively reduced alpha population.

TRANSP and AsCOT simulations have been carried out to predict the fast ion distribution function due to the N-NBI when it is aimed both on- and off-axis, as well as the corresponding pressure and current density profiles [50]. The off-axis fast ion population is peaked at approximately $r / a=0.22$. This fast ion population is strongly passing. The current driven by the neutral beams results in the $q=1$ surface being slightly closer to the magnetic axis than when on-axis NBI is applied.

\subsection{Ion cyclotron resonance heating}

Ion cyclotron waves will be strongly damped in ITER, leading to various possible heating schemes: Second harmonic tritium or fundamental ${ }^{3} \mathrm{He}$ heating; Fundamental D heating (although a low deuterium concentration is required for efficient wave damping making this low relevance); electron Landau damping (ELD)/transit time magnetic pumping (TTMP), both of which are fast wave current drive scenarios leading to insignificant fast ion populations; or off-axis ${ }^{3} \mathrm{He}$ heating, which has moderate absorbed power density but can result in a significant population of ICRH energetic ions near the $q=1$ surface.

The application of ${ }^{3} \mathrm{He}$ minority heating in baseline scenario with the resonance on-axis, slightly off-axis and near mid-radius have been simulated for a range of different minority concentrations. The phasing of the antenna has also been investigated, and it is found that the inward pinch with $+90^{\circ}$ phasing strongly enhances the on-axis fast ion pressure. For the case with $20 \mathrm{MW}$ injected on-axis and minority concentration of $n^{{ }^{3} \mathrm{He}} / n=0.01$ simulated with SELFO, around $70 \%$ of the power absorbed goes into heating the ${ }^{3} \mathrm{He}$ ions (around $7 \mathrm{MW}$ ). The off-axis resonance has also been simulated in order to generate a strong radial gradient in the asymmetry of the passing fast ion distribution near the $q=1$ surface necessary for sawtooth destabilization. Whilst the far-off-axis heating only gives rise to a low power per particle and no highly energetic tails in the distribution, it does nonetheless incur fast ion distributions capable of affecting internal kink stability.

The orbit width effects upon which the internal kink destabilization mechanism are predicated [10] are much smaller in ITER than in JET. In ITER, with ${ }^{3} \mathrm{He}$ minority heating at $52 \mathrm{MHz}$ (i.e. resonance $0.16 \mathrm{~m}$ from the magnetic axis) and toroidal field $B_{\mathrm{T}}=5.3 \mathrm{~T}, 1 \mathrm{MeV}$ 

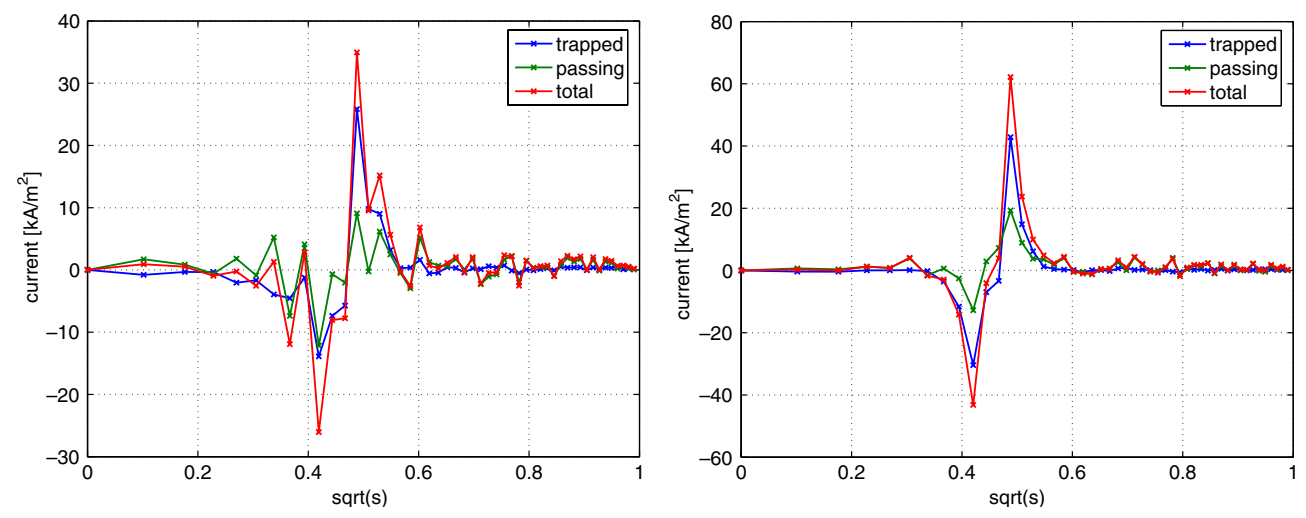

Figure 2. The fast ion currents arising from ICRH of ${ }^{3} \mathrm{He}$ minority near the $q=1$ surface on the low-field side for $0.5 \%{ }^{3} \mathrm{He}$ concentration and (left) $-90^{\circ}$ phasing and (right) $+90^{\circ}$ phasing predicted by SCENIC.

ions have an orbit width $\Delta_{r} / a=0.06$, whereas for comparison, $1 \mathrm{MeV}$ ions in JET with ${ }^{3} \mathrm{He}$ minority at $B_{\mathrm{T}}=2.75 \mathrm{~T}$ (as per experiments in [15]) have an orbit width of $\Delta_{r} / a=0.25$. The fast ion effects are the only way in which the ICRH can contribute to internal kink stability since the strong electron drag means that the change in the magnetic shear due to ICCD will be negligible.

Figure 2 shows the passing and trapped contributions of the deduced flux-averaged fast ion current density predicted by SCENIC as a function of minor radius for ITER at full magnetic field when the ICRH frequency is chosen so that the resonance layer is on the low-field side near the $q=1$ surface. The current drive predicted by SELFo is smaller than in ScENIC and the passing current in SELFO and SCENIC is different. This may be due to differences in the trappedpassing boundary (the boundary between deeply trapped orbits and passing orbits near low-field side stagnation) in SELFO and SCENIC, such that some passing orbits in SCENIC are counted as trapped in SELFo. Also, the precession of deeply trapped ions and stagnation passing ions is different.

The fast ion pressure arising from the ICRH predicted by SELFO is illustrated in figure 3. It is clear that the absorption and the fast ion pressures are reduced by nearly an order of magnitude when the input power is halved. The power partitions for the low-field side off-axis resonance are given in table 1 and compared with the results from AORSA/CQL3D in table 2. The effects of the ICRH fast ions on the internal kink mode stability are discussed in section 5.3. It transpires that the destabilizing effect from the fast ions is least pronounced for the energetic species distribution produced by SELFO, so this is used for the power requirements assessment (hence the scan in power in the SELFo runs).

\section{Effect of energetic particles on sawtooth stability}

\subsection{Alpha particles}

The ITER baseline equilibrium with a monotonic $q$-profile with $q_{0}=0.95, r_{1}=0.42 a$, $q_{95}=3.12$ and $\beta_{p}=0.68$ is found to be unstable to an $n / m=1 / 1$ internal kink mode. The presence of the very energetic alphas inside the $q=1$ position leads to a strong stabilizing effect on the internal kink mode. Figure 4 illustrates the change in the potential energy of the 


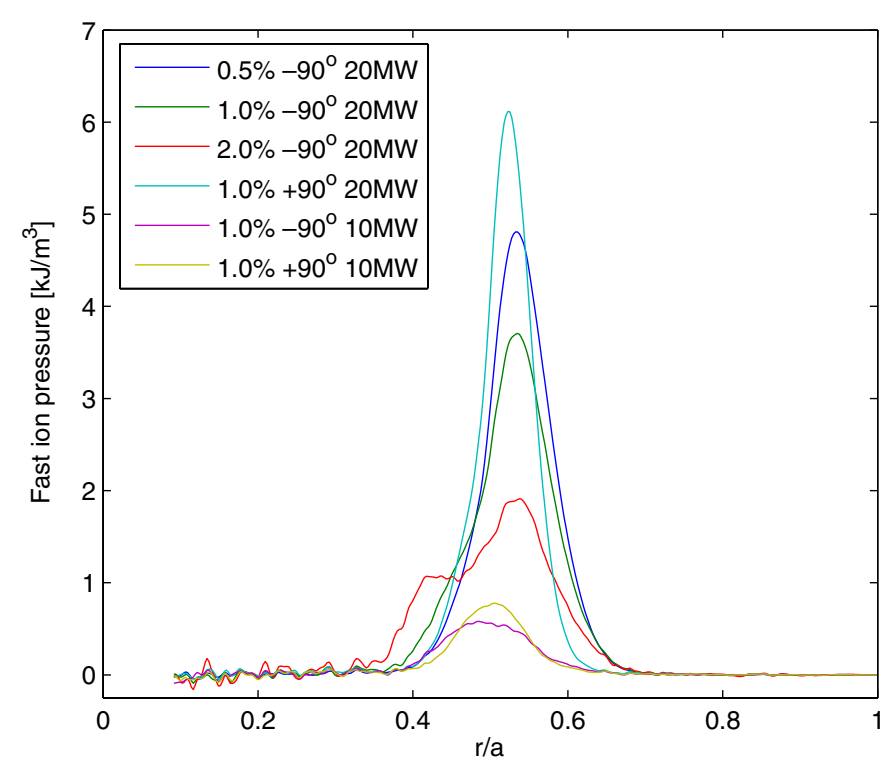

Figure 3. The fast ion pressure arising from ICRH of ${ }^{3} \mathrm{He}$ minority near the $q=1$ surface on the low-field side for different minority concentrations predicted by SELFO.

Table 1. Power partition for off-axis ${ }^{3} \mathrm{He}$ minority ICRH.

\begin{tabular}{lllllll}
\hline${ }^{3}$ He concentration & Antenna phasing & Power & Electrons & ${ }^{3} \mathrm{He}$ & $\mathrm{T}$ & $\mathrm{D}$ \\
\hline $0.5 \%$ & $-90^{\circ}$ & $20 \mathrm{MW}$ & 0.2 & 0.49 & 0.23 & 0.07 \\
$1.0 \%$ & $-90^{\circ}$ & $20 \mathrm{MW}$ & 0.13 & 0.69 & 0.15 & 0.03 \\
$2.0 \%$ & $-90^{\circ}$ & $20 \mathrm{MW}$ & 0.09 & 0.82 & 0.08 & 0.01 \\
$1.0 \%$ & $+90^{\circ}$ & $20 \mathrm{MW}$ & 0.13 & 0.71 & 0.13 & 0.03 \\
$1.0 \%$ & $-90^{\circ}$ & $10 \mathrm{MW}$ & 0.15 & 0.67 & 0.15 & 0.03 \\
$1.0 \%$ & $+90^{\circ}$ & $10 \mathrm{MW}$ & 0.14 & 0.69 & 0.14 & 0.03 \\
\hline
\end{tabular}

kink mode, compared with the fluid drive of the mode. The safety factor of the equilibrium at the magnetic axis has been scaled, and accordingly the radius of $q=1$ has been moved with respect to the deposition location of the off-axis fast ion population. This huge stabilizing contribution from the alpha particles means that without using the heating systems as control actuators, linear theory predicts that the plasma will be very stable to sawteeth. Indeed, the very flat $q$-profile in ITER means that the unmitigated sawtooth period is likely to be extremely long as the current diffusion into the core will take a long time before the increase in the magnetic shear at $q=1$ will cause a sawtooth crash according to the criterion outlined in section 3 . Figure 4 illustrates that the normalized $\delta W$ is almost an order of magnitude larger than the threshold under which the Porcelli model [1] suggests a crash would occur. These simulations demonstrate that the alpha particles are most stabilizing when the $q=1$ surface approaches mid-radius, as expected from transport simulations of the baseline ITER operating scenario. This arises due to the optimization of the average of $\nabla P_{\alpha}$ in the volume of the internal kink displacement. As $r_{1}$ is scanned towards the axis, whilst the gradient of the alpha pressure may increase across $q=1$, the total alpha pressure decreases, meaning that the alpha stabilization is maximized at $r_{1}=0.39 a$, as seen in figure 4 . 
Table 2. Power partition for $20 \mathrm{MW}$ off-axis $1 \%{ }^{3} \mathrm{He}$ minority ICRH with $-90^{\circ}$ antenna phasing as predicted by SELFO and AORSA/CQL3D.

\begin{tabular}{llcll}
\hline Code & Electrons & ${ }^{3} \mathrm{He}$ & $\mathrm{T}$ & $\mathrm{D}$ \\
\hline SELFO & 0.13 & 0.69 & 0.15 & 0.03 \\
AorsA/CQL3D & 0.12 & 0.60 & 0.26 & 0.02 \\
\hline
\end{tabular}

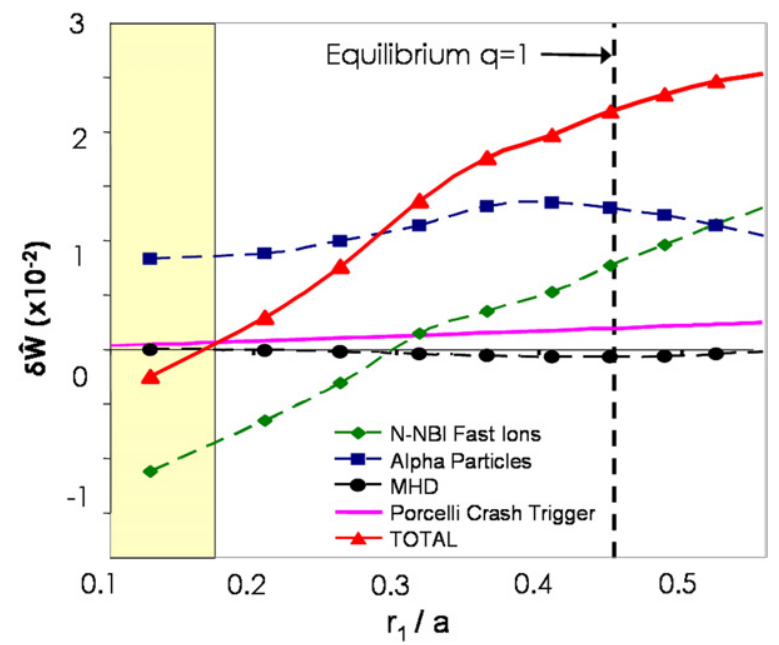

Figure 4. The contributions to the potential energy of the internal kink mode from the presence of the alpha particles, the NBI fast ions and the fluid drive as a function of the minor radius. The shaded region indicates where the linear instability condition given by equation (1) is satisfied, that is to say, when one might expect a sawtooth crash to occur.

\subsection{Neutral beam injection}

The distribution of beam ions produced by both TRANSP and Ascot have been used as input to HAGIS in order to test the effect of the energetic beam ions on internal kink stability. The distribution of beam ions produced by AscoT produced an almost identical level of stabilization as predicted by HAGIS when using the TRANSP distribution, hence alleviating some uncertainty in the prediction by having two independent codes producing similar results. Figure 4 shows the change in the potential energy of the mode including the effects of the passing beam ions in the case when the beam is oriented off-axis with $1 \mathrm{MeV}$ energy and $33 \mathrm{MW}$ power as modelled by TRAnsP. If the peak of the NBI fast ion population is inside the $q=1$ surface, then the long-period sawteeth will be exacerbated. However, if the fast ions are deposited outside $q=1$ then the passing ions have a strongly destabilizing effect on the internal kink. As shown in figure 4, the off-axis N-NBI can destabilize the kink mode sufficiently to cause a sawtooth crash according to the Porcelli model [1] when the $q=1$ surface is within $r / a \approx 0.2$. However, AsTRA predicts that the $q=1$ surface in typical ITER baseline scenario plasmas will be at $r / a \approx 0.45$, as indicated in figure 4 . This implies that without ancillary current drive to move the $q=1$ surface towards the axis, the N-NBI ions will also provide a stabilizing effect on the $n=1$ internal kink mode. Simulations using Asсот have also been performed for the case when the beam energy is $800 \mathrm{keV}$. The stabilizing contribution from the NBI ions drops by $35 \%$ in this case. 


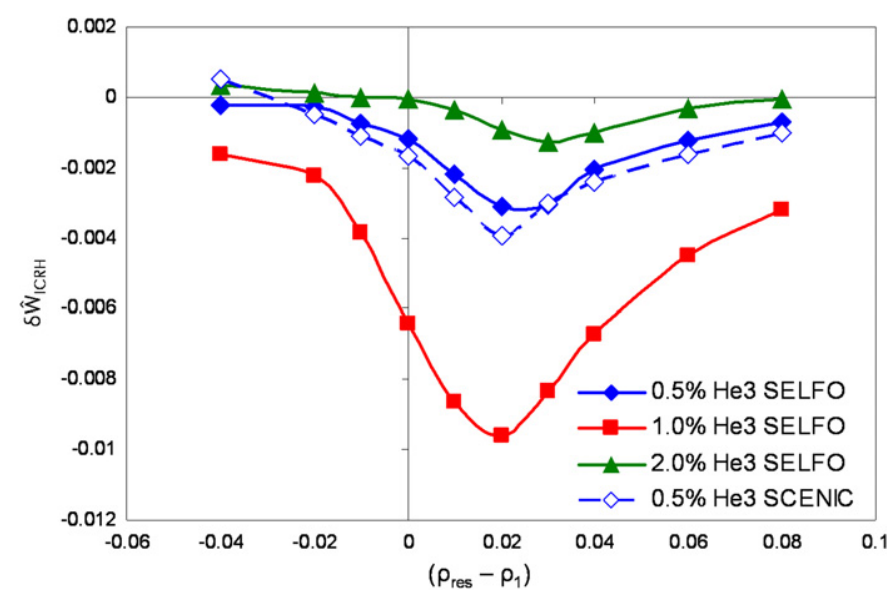

Figure 5. A comparison of $\delta W_{\text {ICRH }}$ predicted by HAGIS using distributions of markers from both SELFO and SCENIC for a case with $0.5 \%{ }^{3} \mathrm{He},-90^{\circ}$ antenna phasing and $20 \mathrm{MW}$ injected power with the resonance at $R=7.284 \mathrm{~m}$. Also shown are results for $1 \%$ and $2 \%$ minority concentration produced by SELFO, showing that $1 \%$ has most effect on the internal kink mode.

\subsection{Ion cyclotron resonance heating}

The applicability of ICRH as a control tool is inferred from HAGIS simulations by considering the ratio of $\delta W_{\text {ICRH }}$ to the contributions from the $\alpha \mathrm{s}$, NBI and fluid drive as a guide to its efficacy. Applying the ICRH off-axis means deposition at lower temperature and therefore shorter slowing down, which makes it more difficult to generate as many fast particles. This means that there are no very energetic tails in the distribution, and the absence of very fast $(>10 \mathrm{MeV})$ particles means that the finite orbit width effects are diminished. That said, however, the ICRH ions do still have a relatively strong impact on the internal kink stability. Figure 5 shows the change in the potential energy of the mode arising due to the ICRH energetic ions as a function of the difference between the resonance radial location and the radius of the $q=1$ surface. There is a clear narrow well in the potential energy when the RF resonance is just outside the rational surface, that is to say when the gradient of the distribution of energetic passing ions is strong and positive. This narrow region $(\sim 4 \mathrm{~cm})$ in which the sawteeth will be sensitive to the destabilizing influence of the ICRH energetic ions-which corresponds to the region in which $\partial j_{\phi} / \partial \psi>0$ in figure 2-implies that real-time control will be required in order that the resonance location be held in the right location with respect to the $q=1$ surface. Despite the fact that the power absorbed by the minority species increases with the concentration, as seen in table 1 , the strongest effect on mode stability is for a ${ }^{3} \mathrm{He}$ concentration of only $1 \%$. When there is too much ${ }^{3} \mathrm{He}$, the energy of the particles in the tail of the distribution becomes too low to have a strong effect on the kink mode, whereas too little ${ }^{3} \mathrm{He}$ means that the absorbed power is low and the broader distribution function leads to increased fast ion losses.

A comparison of $\delta W_{\text {ICRH }}$ when the distribution of markers is taken from SELFO and SCENIC is shown in figure 5 for the case with $0.5 \%{ }^{3} \mathrm{He},-90^{\circ}$ antenna phasing and $20 \mathrm{MW}$ injected power with the resonance at $R=7.284 \mathrm{~m}(f=48.58 \mathrm{MHz}$ in SELFO and $f=48.9 \mathrm{MHz}$ in SCENIC). Whilst the agreement is very good, it is clear that the weakest destabilization is observed using the SELFo distribution. This could be due to the neglect of shaping effects. The main purpose of this comparison, though, is to mitigate the risk in the modelling uncertainty by taking the predictions from the least favourable result, in this case, the SELFo distribution. 


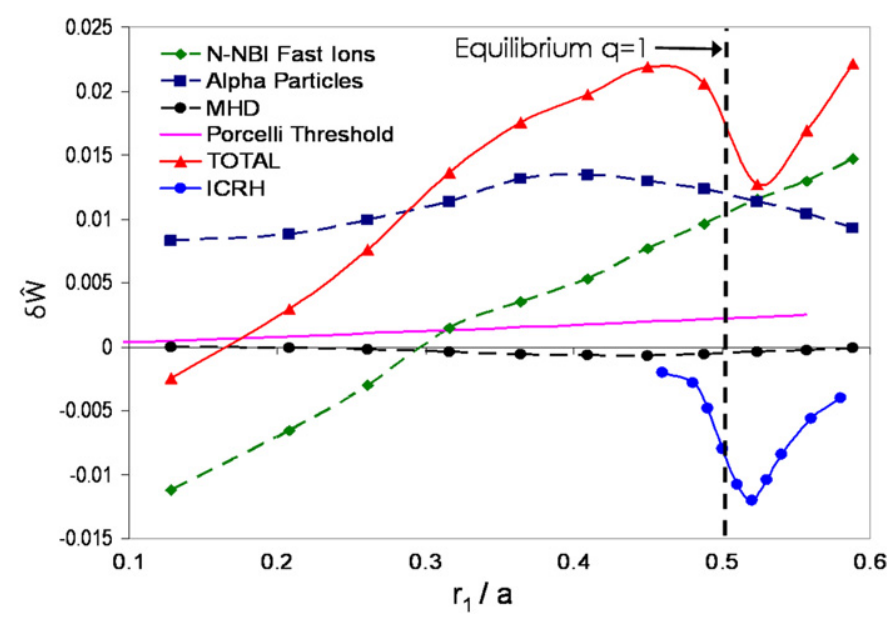

Figure 6. The potential energy of the mode in the presence of all the competing fast ion distributions. It is seen that ICRH significantly destabilizes the mode, despite the strongly stabilizing contribution from the $\alpha$ s and the NNBI ions. Here, the $20 \mathrm{MW}$ of ICRH with $f=48.6 \mathrm{MHz}$ is simulated by SELFo, the $33 \mathrm{MW}$ of off-axis NNBI with Ascot and the alphas with Ascot.

The influence of the ICRH fast ions compared with the stabilizing effect of the alpha particles and NNBI distributions is shown in figure 6 for the case when the ICRH resonance is near the $q=1$ surface. In these simulations the $q=1$ surface is moved by changing the equilibrium rather than re-simulating the fast ion distribution for different resonance locations. It is evident that the mid-radius ICRH fast ions, despite the poor power absorption and low energy tails, retain a strongly destabilizing influence, comparable to the magnitude of stabilization afforded by the alphas or the NBI heating. Whilst the power absorption is better when the resonance layer is nearer to the axis, resulting in improved core heating, the passing fast ions are only destabilizing when the radial location of the $q=1$ surface is inside the deposition radius. These simulations are for $1 \%{ }^{3} \mathrm{He}$ concentration and $+90^{\circ}$ phasing of the antenna, though the $-90^{\circ}$ phasing gives similar results, with a slightly diminished destabilization. The fact that the ICRH is able to completely negate the stabilization from the presence of the $\alpha$ population is significant and important, and suggests that ICRH should be an essential part of the portfolio of control tools in ITER.

Having reduced the risk in the uncertainty of the ICRH fast ion distribution by utilizing independent RF wave field codes, the largest residual uncertainty in this modelling is the location of the $q=1$ surface. The ITER baseline scenario designed using AsTRA transport simulations [52] suggests that the $q=1$ surface will approach mid-radius. However, the $q$-profile has a wide region of very low shear in the core, meaning that a small change in $q_{0}$ can significantly affect the radial location of the rational surface. If the $q=1$ surface could be maintained closer to the magnetic axis, sawtooth control would be significantly easier to achieve, since the alphas and NNBI would be less stabilizing (as illustrated in figure 4) and the control and flexibility afforded by the ICRH would be increased. Furthermore, the ECCD used to control the sawteeth would be closer to the plasma core, and so have the dual benefit of heating on-axis, hence affording a potential reduction in other auxiliary heating power and subsequent increase in $Q$. This may be possible with early heating to delay the current penetration into the core and then deliberate sawtooth destabilization to mediate the $q$-profile once the $q=1$ surface enters. 
Since there is also concern that $20 \mathrm{MW}$ of ICRH power is unlikely to be dedicated for sawtooth control during H-mode operation, the control of the sawteeth with lower powers has also been considered. SELFo simulations of the ICRH fast ion population from $10 \mathrm{MW}$ of ICRH with $1 \%{ }^{3} \mathrm{He}$ minority concentration, $\pm 90^{\circ}$ antenna phasing and $f_{\mathrm{ICRH}}=48.6 \mathrm{MHz}$ have been performed and used as input for HAGIS. The change in the potential energy of the mode at the optimal relative position of $q=1$ with respect to the ICRH resonance location scales approximately linearly with the applied power, despite poor wave absorption. Whilst it is not possible to infer the sawtooth period resultant from the ICRH application from this linear modelling, it is clear that $10 \mathrm{MW}$ of RF heating does significantly destabilize the kink mode, meaning that it is likely to be useful as an ancillary control actuator, even with half the available power. This is supported by empirical evidence from recent JET experiments demonstrating sawtooth control with low RF power and trace ${ }^{3} \mathrm{He}$ minority schemes [15].

\section{Discussion}

Due to the inherent uncertainties in the numerical predictions, it is prudent that a combination of both ICRH and ECCD be considered to control the sawteeth in ITER. It will be necessary to have real-time control of these actuators due to both the uncertainties in the control parameters (launcher aiming, ray-tracing predictions for the driven current, RF frequency) and the equilibrium (plasma position, $q$ profile etc) and the acute sensitivity of the radial location of the heating or current drive with respect to the rational surface. Fortunately, variable frequency ICRH is currently planned for ITER. The ICRH frequency will be able to vary in real-time between $40-55 \mathrm{MHz}$ [53]. The ${ }^{3} \mathrm{He}$ resonance just outside $q=1$ is likely to require a frequency of approximately $47 \mathrm{MHz}$. It will be possible to rapidly change the RF frequency in a preset $2 \mathrm{MHz}$ band, which is equivalent to moving the resonance location by approximately $20 \mathrm{~cm}$, which should provide sufficient scope for realtime sawtooth control. Future experiments should also demonstrate that sawtooth control with active feedback schemes can be successfully applied. This has already been achieved with ECCD, for example $[23,54]$ and initial studies have begun for real-time ICRH control in JET [55].

There are naturally large uncertainties in this modelling, and it is prudent to plan to use more than one control actuator in order to reduce this risk. Consequently, it is recommended that the capability of applying $>10 \mathrm{MW}$ of ICRH at $\sim 47 \mathrm{MHz}$ (with real-time feedback) just outside $q=1$ is retained for sawtooth control to complement $>10 \mathrm{MW}$ of ECCD inside $q=1$ [21]. By reducing the potential energy of the internal kink mode the ICRH not only destabilizes the sawteeth, but also enhances the efficacy of the ECCD control actuator. The largest uncertainty in the modelling of the effect of the fast ions is the position of the $q=1$ surface. If the $q=1$ surface could be maintained closer to the magnetic axis, sawtooth control would be significantly easier to achieve, since both the alphas and the beam-induced fast ions would be less stabilizing. Finally, should active sawtooth destabilization prove to be unattainable due to unexpectedly large stabilizing contribution from the $\alpha$ particles, plant availability or inefficiency in power absorption or current drive, then there is a viable alternative strategy relying upon sawtooth stabilization coupled with pre-emptive NTM suppression [56]. The power requirements for the necessary degree of sawtooth control using either destabilization and stabilization schemes are expected to be within the specification of anticipated ICRH and ECRH heating in ITER, provided the requisite power can be dedicated to sawtooth control. 


\section{Acknowledgments}

This work was partly funded by the RCUK Energy Programme under grant EP/I501045, the Swiss National Science Foundation, the European Communities under the contract of Association between EURATOM and CCFE and US Department of Energy under DE-FC0201ER54648, DE-AC05-00OR22725 and DE-FC02-08ER54952. The views and opinions expressed herein do not necessarily reflect those of the European Commission.

Euratom @ 2011.

\section{References}

[1] Porcelli F, Boucher D and Rosenbluth M 1996 Plasma Phys. Control. Fusion 382163

[2] Chapman I T et al 2007 Plasma Phys. Control. Fusion 49 B385

[3] Hu B, Betti R and Manickam J 2006 Phys. Plasmas 13112505

[4] Sauter O et al 2002 Phys. Rev. Lett. 88105001

[5] Buttery R J et al 2004 20th IAEA Fusion Energy Conf. (Villamoura, Portugal) EX/7-1

[6] Chapman I T et al 2010 Nucl Fusion 50102001

[7] Chapman I T 2011 Plasma Phys. Control. Fusion 53003001

[8] Graves J P et al 2010 Phys. Plasmas 17056118

[9] Graves J P 2004 Phys. Rev. Lett. 92185003

[10] Graves J P et al 2009 Phys. Rev. Lett. 102065005

[11] Chapman I T et al 2007 Phys. Plasmas 14070703

[12] Chapman I T et al 2008 Plasma Phys. Control. Fusion 50045006

[13] Chapman I T et al 2009 Nucl. Fusion 49035006

[14] Chapman I T et al 2009 Phys. Plasmas 16072506

[15] Graves J P et al 2010 Nucl. Fusion. 50052002

[16] Graves J P et al 2011 Fusion Sci. Technol. 59539

[17] Laxaback M and Hellsten T 2005 Nucl. Fusion 451510

[18] Waltz R et al 1997 Phys. Plasmas 42482

[19] Onjun T and Pianroj Y 2009 Nucl. Fusion 49075003

[20] Budny R V et al 2008 Nucl. Fusion 48075005

[21] Zucca C et al 2008 Theory of Fusion Plasmas, Joint Varenna-Lausanne Theory Conf. vol 1069 p 361

[22] Zucca C 2009 Modeling and control of the current density profile in tokamaks and its relation to electron transport PhD Thesis No 4360, EPFL, Lausanne

[23] Lennholm M et al 2009 Phys. Rev. Lett. 102115004

[24] Igochine V et al 2011 Plasma Phys. Control. Fusion 53022002

[25] Budny R V 1992 Nucl. Fusion 32429

[26] Heikkinen J A and Sipila S K 1995 Phys. Plasmas 23724

[27] Kurki-Suonio T et al 2009 Nucl. Fusion 49095001

[28] Hedin J et al 2002 Nucl. Fusion 42527

[29] Jucker M et al 2011 Comput. Phys. Commun. 182912

[30] Jucker M et al 2011 Plasma Phys. Control. Fusion 53054010

[31] Jaeger E F et al 2006 Phys. Plasmas 13056101

[32] Harvey R and McCoy M 1992 Proc. IAEA Technical Committee Meeting on Numerical Modelling of Plasmas (Montreal, Canada) (Vienna: IAEA)

[33] Pinches S D et al 1998 Comput. Phys. Commun. 111133

[34] Suzuki S et al 1998 Plasma Phys. Control. Fusion 402097

[35] Villard L, Brunner S and Vaclavik J 1995 Nucl. Fusion 351173

[36] Carlsson J, Eriksson L-G and Hellsten T 1997 Nucl. Fusion 37719

[37] Cooper W A et al 2006 Nucl. Fusion 46683

[38] Popovich P, Cooper W A and Villard L 2006 Comput. Phys. Commun. 175250

[39] Fischer O, Cooper W A, Isaev M Y and Villard L 2002 Nucl. Fusion 42817

[40] Cooper G A et al 2007 Phys. Plasmas 14102506

[41] Budny R V et al 2010 Proc, 23rd IAEA Fusion Energy Conf. (Daejon, Korea) ITR/P1-29

[42] Porcelli F 1991 Plasma Phys. Control. Fusion 331601

[43] Kruskal M and Oberman C 1958 Phys. Fluids 1275 
[44] Sauter O et al 1998 Theory of Fusion Plasmas, Proc Joint Varenna-Lausanne International Workshop (Varenna, Italy) (New York: AIP) p 403

[45] Bussac M N et al 1975 Phys. Rev. Lett. 351638

[46] Huysmans G T A et al 1991 Proc. CP90 Conf. on Computational Physics (Amsterdam) p 371

[47] Chapman I T, Huysmans G T A, Mikhailovskii A B and Sharapov S E 2006 Phys. Plasmas 13062511

[48] Nave M F F et al 2002 Nucl. Fusion 42281

[49] Asunta O et al http://users.tkk.fi/ tkurki/RIPLOS_FR_Nov2008.pdf

[50] Budny R V 2002 Nucl. Fusion 421383

[51] ITER Technical Basis for Final Design 2001 ITER Documentation Series \#24 (Vienna: IAEA) p 2 chapter 2.5

[52] Gribov Y, ITER Organisation 2010 private communication

[53] Lamalle P, ITER Organisation 2010 private communication

[54] Paley J I et al 2009 Plasma Phys. Control. Fusion 51055010

[55] Lennholm M et al 2011 Nucl. Fusion 51073032

[56] Sauter O et al 2010 Plasma Phys. Control. Fusion 52025002 